\title{
Structural basis for the activation and ligand recognition of the human oxytocin receptor
}

Yann Waltenspühl ${ }^{1}$, Janosch Ehrenmann ${ }^{1,2} \dagger$, Santiago Vacca ${ }^{1} \uparrow$, Cristian Thom $^{1} \uparrow$, Ohad Medalia $^{1}$ and Andreas Plückthun ${ }^{1}$.

${ }^{1}$ Department of Biochemistry, University of Zürich, Winterthurerstrasse 190, CH-8057 Zürich, Switzerland.

${ }^{2}$ Present address: leadXpro AG, PARK innovAARE, CH-5234 Villigen, Switzerland

Correspondence and requests for materials should be addressed to A.P. (plueckthun@,bioc.uzh.ch)

†These authors contributed equally to this work. 


\begin{abstract}
The small cyclic neuropeptide hormone oxytocin (OT) and its cognate receptor play a central role in the regulation of social behaviour and sexual reproduction. Here we report the singleparticle cryo-electron microscopy structure of the active oxytocin receptor (OTR) in complex with its cognate ligand oxytocin. Our structure provides high-resolution insights into the OT binding mode, the OTR activation mechanism as well as the subtype specificity within the oxytocin/vasopressin receptor family.
\end{abstract}




\section{Main}

The neurohypophyseal hormones oxytocin (OT) and arginine vasopressin (AVP) are cyclic peptides that activate an evolutionary ancient subfamily of class A G protein-coupled receptors $(\mathrm{GPCRs})^{1}$, comprising the oxytocin receptor (OTR) and the closely related vasopressin receptors $\left(\mathrm{V}_{1 \mathrm{~A}} \mathrm{R}, \mathrm{V}_{1 \mathrm{~B}} \mathrm{R}\right.$ and $\left.\mathrm{V}_{2} \mathrm{R}\right)$. This $\mathrm{OT} / \mathrm{AVP}$ hormone system is highly conserved among many species and exerts a central role in the regulation of social cognition, social behaviour, and sexual reproduction ${ }^{2}$.

Currently, several clinical trials are evaluating the efficacy of OTR-mediated signalling through administration of OT to treat malfunctions such as autism-spectrum disorders ${ }^{3}$, anxiety ${ }^{4}$ and schizophrenia ${ }^{5}$. While OT itself is an approved peripheral drug in obstetrics ${ }^{6}$, OT-based treatments of socio-behavioural deficiencies, requiring central administration of the hormone, have yet failed, potentially due to its poor drug-like properties ${ }^{7}$ and limited penetration through the blood brain barrier ${ }^{8}$. Despite the high demand for such drugs, the identification and development of OTR-specific molecules with satisfactory pharmacokinetic properties and favourable biodistribution has been impeded by the lack of structural information on the OTR:OT signalling complex. Here, we now report the single-particle cryo-electron microscopy (cryo-EM) structure of the OT-bound human OTR in complex with a heterotrimeric G protein at a resolution of $3.2 \AA$.

Initially, structural studies of the OTR:OT signalling complex were hampered by the poor biophysical behaviour of the wild-type OTR (wtOTR). To improve expression and purification yields we included a single stabilizing mutation (D153Y), which we previously identified by a next-generation sequencing (NGS)-based in-depth analysis of directed evolution experiments ${ }^{9}$. This mutation enabled a 50 -fold increase in the yield of purified functional receptor (Supplementary Fig. 1), with very similar agonist binding and signalling behaviour. As an additional hurdle, complexes of OTR with an otherwise frequently employed engineered 
mini- $\mathrm{G}_{\mathrm{s}}{ }^{10,11}$ or a $\mathrm{G}_{\mathrm{s} / \mathrm{q}}$ chimera were not stable and dissociated upon plunge freezing. While $\mathrm{G}_{\mathrm{q}^{-}}$ based signalling is the main route of OTR activation, the receptor has been shown to also interact with $\mathrm{G}_{\mathrm{o}}$ and $\mathrm{G}_{\mathrm{i}}{ }^{12}$, but not with $\mathrm{G}_{\mathrm{s}}$. Therefore, we hypothesized that the observed instability of the OTR:mini-G $\mathrm{G}_{\mathrm{s} / \mathrm{q}}$ complex may be attributed to unfavourable interactions of OTR with the $\mathrm{G}_{\mathrm{s}}$ domain, and interactions of the $\mathrm{G}_{\mathrm{q}} \alpha 5$ helix are not sufficient to overcome this. Thus, to maximally stabilize the OTR active state, we designed a new G protein chimera using mini- $\mathrm{G}_{\mathrm{o}}{ }^{10}$ as a basis, substituting the $\mathrm{G}_{\mathrm{o}} \alpha 5$ helix with the corresponding amino acids of $\mathrm{G}_{\mathrm{q}}$. Additionally, we replaced the $\mathrm{N}$ terminus with the respective $\mathrm{G}_{\mathrm{i} 1}$ residues to permit binding of the complex-stabilizing single-chain variable fragment 16 (scFv16). For simplicity, the resulting mini-G protein is denoted $\mathrm{G}_{\mathrm{o} / \mathrm{q}}$ henceforth. These combined engineering efforts finally enabled single-particle cryo-EM analysis of the OTR:OT:G $\mathrm{G}_{\mathrm{o} / \mathrm{q}}: \mathrm{ScFv} 16$ complex at a resolution of $3.2 \AA$ (Fig. 1a,b, Supplementary Figs. $2-4$ and Supplementary Table 1), enabling unprecedented insights into OTR activation by OT and the receptor G protein interaction.

In the orthosteric ligand binding pocket, all nine amino acids of OT participate in OTR binding (Fig. 1c). The cyclic part (residues $1-6$ ) is buried deep inside the pocket while the Cterminal tripeptide (residues 7 - 9) is facing the extracellular loops. Interestingly, the amidated C-terminus of Gly ${ }^{9}$, known to be important for activation ${ }^{13}$, is located in the proximity of residues E42 ${ }^{1.35}$ and D100 2.65 (numbers in superscripts correspond to Ballesteros-Weinstein numbering) of transmembrane helices I and II, which are involved in magnesium-dependent modulation of OT binding ${ }^{14}$. Together with the neighbouring $\mathrm{Leu}^{8}$, the extracellular surface of the orthosteric pocket is lined by $\operatorname{Pro}^{7}$ and $\mathrm{Asn}^{5}$, which pack against extracellular loop 3 (ECL3) and ECL2, respectively. $\mathrm{Leu}^{8}$ is oriented towards the extracellular space, explaining why position 8 is best suited for the attachment of fluorophores in $\mathrm{OT}^{15}$. $\mathrm{Gln}^{4}$ is the only residue pointing out perpendicularly from the ring plane and stabilizes the cyclic ring position through a hydrogen bond to $\mathrm{Q} 295^{6.55}$. $\mathrm{Ile}^{3}$ is buried in a hydrophobic pocket formed by side-chain 
residues of transmembrane helices IV, V, and VI. The critical contribution of this hydrophobic pocket is underlined by the observed reduction in OT potency when mutating the main contact residues I201 ${ }^{5.39}$, I204 ${ }^{5.42}$ and F291 ${ }^{6.51}$ of the receptor to alanine (Fig. 1d,e and Supplementary Table 2). $\operatorname{Tyr}^{2}$ penetrates deep into a crevice at the bottom of the orthosteric pocket formed by residues from helices II, III, VI and VII. While the carboxy group of $\mathrm{Tyr}^{2}$ interacts with Q171 ${ }^{4.60}$, the phenol ring engages in hydrophobic interactions with Q92 2.57 and F291 ${ }^{6.51}$, and the hydroxyl group forms a hydrogen bond to the backbone amide oxygen of L316 7.40 . The importance of these interactions explains the loss of potency when either Q171 ${ }^{4.60}$ or F291 ${ }^{6.51}$ are mutated to alanine (Fig. 1d,e). Finally, $\mathrm{Cys}^{1}$, which stabilizes the OT ring conformation through a disulphide with $\mathrm{Cys}^{6}$, contacts with its backbone oxygen a polar cluster consisting of residues Q96 ${ }^{2.61}$, K116 3.29 , and Q1193.32 observed in the OT/AVP family. (Fig. 1d,e)

A comparison of the OTR active-state structure with the previously reported inactivestate structure of the OTR in complex with the small-molecule antagonist retosiban ${ }^{14}$ enabled us to identify the molecular changes involved in receptor activation (Fig 2a and Supplementary Fig. 5). In contrast to OT, retosiban only partially occupies the region of the orthosteric pocket, where the cyclic part of OT binds. Nonetheless, the OT-induced helical rearrangements in the orthosteric pocket are relatively small, reflected by the subtle change of the pocket volume between the active and inactive state (Supplementary Fig. 5). OT interacts with residues F291 $1^{6.51}$ and $F 292^{6.52}$ at the bottom of the binding pocket. This interaction induces a rearrangement of F291 ${ }^{6.51}$, initiating the large outward movement of helix VI through a series of side-chain reorientations in conserved microswitches, including W2886.48 (CWxP motif), F284 $4^{6.44}$ (PIF motif) and Y3297.53 (NPxxY motif) (Fig. 2a). These rearrangements ultimately lead to the breakage of the interaction between T273 3.33 and R $137^{3.50}$ of the DRY motif (DRC in OTR), and the reorientation of D136 3.49 into a position enabling direct contact with the $\alpha 5$ helix of $\mathrm{G}_{\mathrm{o} / \mathrm{q}}$ (Supplementary Fig. 5). 
Early functional studies on OT derivatives identified $\mathrm{Cys}^{1}$, $\mathrm{Tyr}^{2}$ and $\mathrm{Gln}^{4}$ as centrally involved ligand residues in receptor activation. For example, alkylation of the $\mathrm{Tyr}^{2}$ hydroxy group led to decreased agonistic activity ${ }^{7}$. Remarkably, we observe a local unfolding of helix VII at the extracellular receptor side in the region of L $316^{7.40}$, creating a pronounced kink which is stabilized by a hydrogen bond formed between $\mathrm{Tyr}^{2}$ of OT with the backbone oxygen of L316 ${ }^{7.40}$ (Fig. 2a). Importantly, a similar helix VII conformation was also observed in activestate structures of $\mathrm{V}_{2} \mathrm{R}^{16}$ (Fig. 2b), suggesting that partial helix VII unfolding is a feature of the OT/AVP family receptor activation. Sequence alignments of the four receptors of the OT/AVP family reveal that all receptors share a conserved kink region, with the exception of position 7.42 where, both OTR and $V_{2} R$ share an alanine, whereas $V_{1 A} R$ and $V_{1 B} R$ carry a glycine. To test if a kink region carrying a glycine is compatible with the observed helix VII reorientation, we determined ligand binding affinity and receptor activation of an OTR mutant where we mutated $\mathrm{A} 318^{7.42}$ to glycine. We observe only little differences in activity and binding affinity, supporting an unaltered activation mechanism. It appears that glycine might potentially destabilize the local helical conformation and even facilitate ligand binding, as indicated by the slightly improved affinity and potency of OT to A31 $8^{7.42} \mathrm{G}$. Accordingly, we find that removal of the glycine in $\mathrm{V}_{1 \mathrm{~A}} \mathrm{R}$ by mutation $\mathrm{G} 337^{7.42} \mathrm{~A}$ has the inverse effect (Supplementary Fig. 6, Supplementary Tables 2 and 3).

OT differs only in two positions from the closely related AVP (Ile ${ }^{3}$ and $\mathrm{Leu}^{8}$ in OT $v s$. $\mathrm{Phe}^{3}$ and $\mathrm{Arg}^{8}$ in AVP) (Supplementary Fig. 7a). While these differences suffice to render OT specific for the OTR over the vasopressin receptors, the OTR itself is not selective between OT and $\mathrm{AVP}^{17}$. To investigate possible contributions to subtype selectivity, we compared the OTbound OTR structure to the previously published AVP-bound $V_{2}$ R structures ${ }^{16,18,19}$ (Fig. 2b and Supplementary Fig. 7b,c). Both OT and AVP adopt a similar orientation in the orthosteric pocket of their respective receptor with highest similarity observed for the agonist's cyclic 
portion, where $\mathrm{Phe}^{3}$ of AVP penetrates only marginally deeper than $\mathrm{Ile}^{3}$ of OT (Fig. 2b). The largest structural differences are found for positions eight and nine of the ligand in the tripeptide C-terminus. Leu ${ }^{8}$ and Gly ${ }^{9}$ in OT are located along the ring plane, with Gly ${ }^{9}$ facing helix I (Figs. $1 \mathrm{~d}$ and $2 \mathrm{~b}$ and Supplementary Fig. 7). Conversely, $\mathrm{Arg}^{8}$ and $\mathrm{Gly}^{9}$ in AVP, which in each reported $V_{2} R$ :AVP structure have been modelled differently, are facing away from the ring plane enabling contacts to residues of ECL1, ECL3 and the N-terminus of $V_{2} R$. In the OTR, helix I adopts a position further away from the central axis of the receptor compared to $V_{2} R$. This wider opening of the orthosteric pocket in OTR enables binding of the OT C-terminal residues $\mathrm{Leu}^{8}$ and Gly ${ }^{9}$ in the observed conformation. In $\mathrm{V}_{2} \mathrm{R}$, however, OT binding would be sterically compromised due to a clash of Gly ${ }^{9}$ with helix I. In contrast, the binding conformation of AVP in $V_{2} R$ is compatible with OTR binding. Therefore, the positioning of helix I might explain why the OTR is not selective between OT and AVP, but OT binding is specific to OTR and not $\mathrm{V}_{2} \mathrm{R}$. In variance to long-standing models ${ }^{17,20}$, we do not observe an interaction of OT with R34 ${ }^{\mathrm{N}-\text { terminus }}$ or F103 ${ }^{\mathrm{ECL} 1}$ of the OTR. This might indicate an allosteric effect, or in the case of R34, which does not show clear density, a more dynamic interaction. We speculate that this could additionally contribute to subtype selectivity in the OT/AVP receptor family.

On the intracellular receptor side, the main conformational differences between OTR and $\mathrm{V}_{2} \mathrm{R}$ are found in the positioning of the helix VII-VIII transition region and the elongation of helix V of $\mathrm{V}_{2} \mathrm{R}$ compared to OTR (Fig 2b). Both regions contribute to G protein binding, and the respective differences are likely a feature of the diverse binding modes between the receptors and the interacting $\mathrm{G} \alpha$ subunits $\left(\mathrm{V}_{2} \mathrm{R}: \mathrm{G}_{\mathrm{s}}\right.$ and $\left.\mathrm{OTR}: \mathrm{G}_{\mathrm{o} / \mathrm{q}}\right)$.

In the signalling complex of the activated OTR, the $\alpha 5$ helix of $\mathrm{G}_{\mathrm{o} / \mathrm{q}}$ is bound in a crevice constituted by helices II, III, V, VI and VII at the receptor intracellular side, where the G protein C-terminal residues E350 and Y351 engage in hydrogen bonding with R73 $3^{2.38}$ and D136 $6^{3.49}$ of OTR (Fig. 2c). Compared to reported receptor: $\mathrm{G}_{\mathrm{s} / \mathrm{q}}$ complexes ${ }^{11,21,22}$, the $\mathrm{G}_{\mathrm{q}} \alpha 5$ helix in 
OTR: $\mathrm{G}_{\mathrm{o} / \mathrm{q}}$ is rotated away from helix VI (Fig. 2d). This rotation cannot be attributed to differences in chimera design, as there is a perfect structural alignment of the individual $\mathrm{G}_{\alpha}$ subunits. Most importantly, the same rotation is also distinct from GPCR: $\mathrm{G}_{\mathrm{o}}$ strcutures $^{23-25}$ (Supplementary Fig. 8). Instead, the orientation of the $\mathrm{G}_{\mathrm{q}} \alpha 5$ helix in OTR: $\mathrm{G}_{\mathrm{o} / \mathrm{q}}$ very much resembles that of $G_{s}$ coupled to $V_{2} R$, with the latter penetrating less deeply into the $V_{2} R$ intracellular TMD crevice, suggesting the receptor is governing the $\alpha 5$ orientation (Fig. 2e).

In conclusion, we report here the first structure of the human OTR:OT signalling complex, providing insights into the subfamily-specific OTR activation mechanism and an unexpected $\mathrm{G}$ protein binding mode as well as the detailed OT binding mode, thereby enhancing our understanding of the subtype specificity within the closely related oxytocin and vasopressin receptor family. Together, these findings are expected to greatly facilitate the development of novel therapeutics for the treatment of a variety of OTR-implicated diseases. 


\section{Material \& Methods}

\section{Design of complex constructs}

The sequences of scFv16 26 and of wild-type human OTR (wtOTR), codon-optimized for expression in Spodoptera frugiperda (Sf9) (C-terminally truncated after residue 359), were cloned into a modified pFL vector (MultiBac system, Geneva Biotech) for Sf9 expression. The resulting expression constructs contained a melittin signal sequence, followed by a FLAG-tag, a His 10 -tag, and a human rhinovirus $3 \mathrm{C}$ protease cleavage site $\mathrm{N}$-terminal to the gene of interest. To increase OTR purification yield, the mutation D153Y was introduced into the truncated wtOTR sequence, as identified previously ${ }^{9}$. It shows very similar KD and EC50 for oxytocin (OT) (Supplementary Fig. 1). The mutant was generated by sequence- and ligation-independent cloning as previously described in detail ${ }^{27}$. To generate an $\mathrm{G}_{\mathrm{o} / \mathrm{q}}$ subunit that would allow interaction with $\mathrm{scFv} 16^{25}$, the N-terminal 18 amino acids of $\mathrm{G}_{\mathrm{i} 1}$ were introduced to the engineered mini- $\mathrm{G}_{01} 12^{10}$. To generate $\mathrm{G}_{\mathrm{q}}$-like interactions, residues H5.16, H5.17, H5.18, H5.22, H5.23, H5.24, and H5.26 (according to the common Ga numbering) ${ }^{28}$ in the C-terminal helix were mutated to corresponding amino acids of $\mathrm{G}_{\mathrm{q}}$. Finally, the engineered $\mathrm{G}_{\mathrm{o} / \mathrm{q}}$ chimera sequence was cloned into one $\mathrm{pFL}$ vector, together with $\mathrm{G} \beta_{1}$ (including an N-terminal noncleavable His 10 -tag) and with $\mathrm{G} \gamma_{2}$, with each gene under the control of its own polyhedrin promoter.

\section{Expression and purification of OTR}

Expression and purification were performed as previously described in detail ${ }^{11,14}$. In brief, $4 \mathrm{~L}$ of $S f 9$ insect cell culture at a density of $3 \times 10^{6}$ cells $/ \mathrm{ml}$ were infected with baculovirus stocks at a multiplicity of infection $\geq 5$. Cells were harvested $72 \mathrm{~h}$ post-infection and stored at $-80^{\circ} \mathrm{C}$. Cells were thawed on ice before purification, lysed, and membranes were isolated by repeated Dounce homogenization in hypotonic buffer containing $10 \mathrm{mM}$ Hepes $(\mathrm{pH} 7.5), 20$ 
$\mathrm{mM} \mathrm{KCl}, 10 \mathrm{mM} \mathrm{MgCl}$, and protease inhibitors $(50 \mu \mathrm{g} / \mathrm{ml}$ Pefabloc SC and $1 \mu \mathrm{g} / \mathrm{ml}$ Pepstatin A, both Carl Roth) and then in hypertonic buffer containing $10 \mathrm{mM}$ Hepes (pH 7.5), $20 \mathrm{mM}$ $\mathrm{KCl}, 10 \mathrm{mM} \mathrm{MgCl} 2,1.0 \mathrm{M} \mathrm{NaCl}, 0.1 \mathrm{mg} / \mathrm{ml}$ deoxyribonuclease (DNase, MilliporeSigma) and protease inhibitors. Washed membranes were resuspended in hypotonic buffer, and the lowaffinity antagonist SSR 149415 (Tocris) was added to a final concentration of $100 \mu \mathrm{M}$ and the suspension was incubated for $30 \mathrm{~min}$. Then, iodoacetamide $(2 \mathrm{mg} / \mathrm{ml}$ final concentration; MilliporeSigma) was added to the solution followed by another $30 \mathrm{~min}$ of incubation. Subsequently, the receptor was solubilized in buffer containing $30 \mathrm{mM}$ Hepes (pH 7.5), 500 $\mathrm{mM} \mathrm{NaCl}, 10 \mathrm{mM} \mathrm{KCl}, 5 \mathrm{mM} \mathrm{MgCl} 2,50 \mu \mathrm{M}$ SSR 149415, 1\% (w/v) n-dodecyl- $\beta$-dmaltopyranoside (DDM, Anatrace), and $0.2 \%(\mathrm{w} / \mathrm{v})$ cholesteryl hemisuccinate (CHS, MilliporeSigma) for 3 hours at $4^{\circ} \mathrm{C}$. Insoluble material was removed by ultracentrifugation at $220,000 \mathrm{~g}$, and the supernatant containing the solubilized receptor was incubated with TALON IMAC resin (Cytiva) at $4^{\circ} \mathrm{C}$.

The receptor-bound resin was washed with 20 column volumes (CVs) of wash buffer I containing $50 \mathrm{mM}$ Hepes (pH 7.5), $500 \mathrm{mM} \mathrm{NaCl}, 10 \mathrm{mM} \mathrm{MgCl}_{2}, 5 \mathrm{mM}$ imidazole, $10 \%$ (v/v) glycerol, $8 \mathrm{mM}$ adenosine triphosphate (ATP, MilliporeSigma), supplemented with $0.5 \%$ (w/v) DDM, 0.01\% (w/v) CHS, and $10 \mu \mathrm{M}$ SSR 149415. Subsequently, the detergent was exchanged with $16 \mathrm{CVs}$ of wash buffer I supplemented with $1 \%(\mathrm{w} / \mathrm{v})$ lauryl maltose neopentyl glycol (LMNG, Anatrace), 0.1\% (w/v) CHS, and $10 \mu \mathrm{M}$ SSR 149415, and the antagonist was replaced with OT (Psyclo Peptide Inc.) with another 16 CVs of wash buffer I supplemented with $0.5 \%$ (w/v) LMNG, 0.05\% (w/v) CHS, and $100 \mu \mathrm{M}$ OT. Agonist-bound OTR was finally washed with $25 \mathrm{CVs}$ of wash buffer II containing $50 \mathrm{mM}$ Hepes (pH 7.5), $150 \mathrm{mM} \mathrm{KCl,} 15 \mathrm{mM}$ imidazole, $10 \%(\mathrm{v} / \mathrm{v})$ glycerol, $0.05 \%(\mathrm{w} / \mathrm{v}) \mathrm{LMNG}, 0.005 \%(\mathrm{w} / \mathrm{v}) \mathrm{CHS}$, and $50 \mu \mathrm{M}$ OT, before eluting the receptor in 4 subsequent fractions of $1 \mathrm{CV}$ of elution buffer containing $50 \mathrm{mM}$ Hepes 
(pH 7.5), $150 \mathrm{mM} \mathrm{KCl,} 250 \mathrm{mM}$ imidazole, $10 \%$ (v/v) glycerol, $0.01 \%$ (w/v) LMNG, $0.001 \%$ (w/v) CHS, and $50 \mu \mathrm{M}$ OT.

Protein-containing fractions were combined and concentrated to $0.5 \mathrm{ml}$ using a $50-\mathrm{kDa}$ molecular weight cutoff (MWCO) Vivaspin 2 concentrator (Sartorius), and desalted by buffer exchange on a PD MiniTrap G-25 column (Cytiva) equilibrated with G25 buffer containing 50 $\mathrm{mM}$ Hepes (pH 7.5), $150 \mathrm{mM} \mathrm{KCl,} \mathrm{10 \%} \mathrm{(v/v)} \mathrm{glycerol,} \mathrm{0.01 \%} \mathrm{(w/v)} \mathrm{LMNG,} 0.001 \%$ (w/v) $\mathrm{CHS}$, and $50 \mu \mathrm{M}$ OT. To remove the $\mathrm{N}$-terminal affinity tags and to deglycosylate the receptor, OT-bound receptor was treated with His-tagged 3C protease and His-tagged peptide Nglycosidase (PNGase) F (both prepared in-house) overnight. To collect cleaved receptor, the reaction was incubated with Ni-nitrilotriacetic acid (Ni-NTA) resin (Cytiva) for $1 \mathrm{~h}$, cleaved receptor was collected as the flow-through, then concentrated to $\sim 3$ to $5 \mathrm{mg} / \mathrm{ml}$ with a $50-\mathrm{kDa}$ MWCO Vivaspin 2 concentrator, and directly used for complex formation. Protein purity and monodispersity were assessed by LDS-polyacrylamide gel electrophoresis and analytical size exclusion chromatography (SEC) using a Nanofilm SEC-250 column (Sepax).

\section{Purification of $\mathbf{G}_{\mathbf{0} / \mathbf{q}}$}

Purification of the engineered heterotrimeric $G$ protein was carried out similarly to receptor purification, with small adaptations. All buffers used were supplemented with $10 \mu \mathrm{M}$ guanosine diphosphate (GTP, MilliporeSigma) and $100 \mu \mathrm{M}$ tris(2-carboxyethyl)phosphine) (TCEP, Thermo Fisher Scientific). In contrast to the receptor purification, monovalent cation concentration never exceeded $150 \mathrm{mM}$, and all buffers were devoid of any receptor ligands and iodoacetamide. Enriched G protein-containing membranes were washed by Dounce homogenization without high salt concentrations in physiological buffer, containing $10 \mathrm{mM}$ Hepes ( $\mathrm{pH}$ 7.5), $150 \mathrm{mM} \mathrm{NaCl}, 20 \mathrm{mM} \mathrm{KCl}, 10 \mathrm{mM} \mathrm{MgCl}_{2}$, and protease inhibitors. Solubilization and immobilization on TALON IMAC resin was performed as described above. 
The G protein-bound resin was initially washed with $30 \mathrm{CVs}$ of wash buffer I containing $50 \mathrm{mM}$ Hepes (pH 7.5), $150 \mathrm{mM} \mathrm{KCl,} 10 \mathrm{mM} \mathrm{MgCl} 2,5 \mathrm{mM}$ imidazole, 10\% (v/v) glycerol, $1 \%(\mathrm{w} / \mathrm{v})$ DDM, $0.2 \%(\mathrm{w} / \mathrm{v})$ CHS, $10 \mu \mathrm{M}$ GTP and $100 \mu \mathrm{M}$ TCEP followed by a wash with 30 $\mathrm{CV}$ of detergent exchange buffer containing $50 \mathrm{mM}$ Hepes (pH 7.5), $150 \mathrm{mM} \mathrm{KCl}, 1 \mathrm{mM}$ $\mathrm{MgCl}_{2}, 5 \mathrm{mM}$ imidazole, 10\% (v/v) glycerol, 1\% (w/v) LMNG, 0. 1\% (w/v) CHS 8 mM ATP, $10 \mu \mathrm{M}$ GTP and $100 \mu \mathrm{M}$ TCEP. G proteins were finally washed with $30 \mathrm{CVs}$ of wash buffer II containing $50 \mathrm{mM}$ Hepes (pH 7.5), $150 \mathrm{mM} \mathrm{KCl,} 1 \mathrm{mM} \mathrm{MgCl} 2,15 \mathrm{mM}$ imidazole, $10 \%$ (v/v) glycerol, $0.01 \%$ (w/v) LMNG, 0.001\% (w/v) CHS, $10 \mu \mathrm{M}$ GTP and $100 \mu \mathrm{M}$ TCEP and eluted stepwise in 4 subsequent fractions of $1 \mathrm{CV}$ of elution buffer containing $750 \mathrm{mM}$ imidazole.

Eluted G protein was concentrated using a 100-kDa MWCO Vivaspin 2 concentrator and further purified using a Superdex 200 10/300 (Cytiva) column equilibrated with SEC buffer containing $25 \mathrm{mM}$ Hepes (pH 7.5), $150 \mathrm{mM} \mathrm{KCl,} \mathrm{2 \%} \mathrm{(v/v)} \mathrm{glycerol,} \mathrm{0.01 \%} \mathrm{(w/v)} \mathrm{LMNG,} \mathrm{and}$ $0.001 \%(\mathrm{w} / \mathrm{v})$ CHS. Fractions containing monodisperse G protein were collected, concentrated to $7.1 \mathrm{mg} / \mathrm{ml}$ with a $100-\mathrm{kDa}$ MWCO Vivaspin 2 concentrator (Sartorius), frozen in liquid nitrogen, and stored at $-80^{\circ} \mathrm{C}$ until use.

\section{Purification of scFv16}

ScFv16 was expressed and purified as described before ${ }^{11}$. Briefly, scFv16 was expressed by secretion from baculovirus-infected $S f 9$ cells for $72 \mathrm{~h}$. ScFv16-containing expression medium was separated from cells by centrifugation, then $\mathrm{pH}$-adjusted by addition of Hepes ( $\mathrm{pH}$ 7.5). Metal-chelating agents from the cells and medium were quenched by incubation with $1 \mathrm{mM} \mathrm{CoCl}_{2}$ and $5 \mathrm{mM} \mathrm{CaCl}_{2}$ for 1 hour at $22^{\circ} \mathrm{C}$. Resulting precipitates were removed by centrifugation, and the filtrated supernatant was loaded onto a $\mathrm{Co}^{2+}$-loaded HiTrap IMAC HP column (Cytiva). The column was washed with $20 \mathrm{CVs}$ of buffer A containing 20 mM Hepes (pH 7.5), $150 \mathrm{mM} \mathrm{NaCl}, 1 \mathrm{mM} \mathrm{MgCl}_{2}, 4 \mathrm{mM}$ ATP, and $5 \mathrm{mM}$ imidazole, followed 
by $20 \mathrm{CVs}$ of buffer B containing $20 \mathrm{mM}$ Hepes (pH 7.5), $150 \mathrm{mM} \mathrm{NaCl}$, and $30 \mathrm{mM}$ imidazole. The protein was eluted with buffer $\mathrm{C}$ [20 mM Hepes (pH 7.5), $150 \mathrm{mM} \mathrm{NaCl}$, and $300 \mathrm{mM}$ imidazole]. Monomeric fractions were pooled, concentrated using a 10-kDa MWCO Amicon Ultra concentrator (Merck), and imidazole was removed by applying the concentrate to a PD10 desalting column (Cytiva) equilibrated with G25-buffer containing $50 \mathrm{mM}$ Hepes (pH 7.5), $150 \mathrm{mM} \mathrm{NaCl}, 10 \%$ (v/v) glycerol, $0.01 \%$ (w/v) LMNG, and 0.001\% (w/v) CHS. ScFv16 was incubated for 3 hours with His-tagged 3C protease and His-tagged PNGase F for removal of affinity tags and deglycosylation. After incubation with Ni-NTA resin for $1 \mathrm{~h}$, cleaved scFv16 was collected as the flow-through, concentrated with a $10-\mathrm{kDa}$ MWCO Amicon Ultra concentrator, and further purified using a Superdex 200 10/300 column equilibrated with SEC buffer. Monomeric fractions were pooled, concentrated to $\sim 6$ to $7 \mathrm{mg} / \mathrm{ml}$, flash-frozen in liquid nitrogen, and stored at $-80^{\circ} \mathrm{C}$. Right before complex formation, scFv16 was thawed and the buffer was exchanged on a PD MiniTrap ${ }^{\mathrm{TM}}$ G-25 column (Cytiva) equilibrated with G25-buffer.

\section{Complex formation}

Purified OTR and engineered heterotrimeric $G$ protein $\left(G \alpha_{o} / q \beta_{1} \gamma_{2}\right)$ were mixed in a molar ratio of 1:1.2 in complexation buffer (25 mM Hepes (pH 7.5), $100 \mathrm{mM} \mathrm{KCl,} 1 \mathrm{mM}$ $\mathrm{MgCl}_{2}, 0.01 \%(\mathrm{w} / \mathrm{v}) \mathrm{LMNG}, 0.001 \%$ (w/v) CHS, $100 \mu \mathrm{M}$ OT, and $100 \mu \mathrm{m}$ TCEP). After 30 minutes, apyrase $(0.8 \mathrm{U} / \mathrm{ml}$; MilliporeSigma) and $\lambda$-phosphatase $(1,000 \mathrm{U} / \mathrm{ml}$, New England Biolabs) were added to the mixture. After $2 \mathrm{~h}$, purified scFv16 was added at 4-fold molar excess over receptor, and complex formation was allowed to proceed overnight at $4^{\circ} \mathrm{C}$. Stable complex was isolated by SEC on a Superdex 200 10/300 column (Cytiva) equilibrated with blotting buffer (25 mM Hepes (pH 7.5), $100 \mathrm{mM} \mathrm{KCl,} 1 \mathrm{mM} \mathrm{MgCl} 2,100 \mu \mathrm{M}$ OT, and $100 \mu \mathrm{m}$ TCEP, $0.001 \%(\mathrm{w} / \mathrm{v}) \mathrm{LMNG}$, and $0.0001 \%(\mathrm{w} / \mathrm{v}) \mathrm{CHS})$. Corresponding peak fractions were 
concentrated to $1 \mathrm{mg} / \mathrm{ml}$ for EM studies, using a 100-kDa MWCO Vivaspin Turbo PES (Sartorius) concentrator.

\section{Single-particle cryo-EM data collection}

For cryo-EM grid preparation, $3 \mu \mathrm{L}$ of purified OTR:OT: $\mathrm{G}_{\mathrm{o} / \mathrm{q}}: \mathrm{scFv} 16$ complex in blotting buffer were applied to glow-discharged holey carbon gold grids (Quantifoil R1.2/1.3, 300 mesh), and subsequently vitrified using a Vitrobot Mark IV (Thermo Fisher Scientific) operated at $100 \%$ humidity and $4{ }^{\circ} \mathrm{C}$. Cryo-EM images were acquired by a Titan Krios G3i electron microscope (Thermo Fisher Scientific), operated at $300 \mathrm{kV}$, at a nominal magnification of 130,000 using a K3 direct electron detector (Gatan) in super-resolution mode, corresponding to a pixel size of $0.325 \AA$. A BioQuantum energy filter (Gatan) was operated in a zero-loss mode, using $20 \mathrm{eV}$ energy slit-width. A total of 6,450 movies were obtained, with a defocus range of -0.8 to $-2.4 \mu \mathrm{m}$ using automatic data acquisition with EPU software (Thermo Fisher Scientific). The total exposure time was $1.79 \mathrm{~s}$ with an accumulated dose of $\sim 63.78$ electrons $/ \AA^{2}$ and a total of 67 frames per micrograph. A second set of 5,217 image stacks were acquired with the same conditions and parameters.

\section{Single-particle cryo-EM data processing}

All image stacks were binned to generate a pixel size of $0.65 \AA$ followed by motioncorrection and dose-weighting using MotionCor $2^{29}$. All images were contrast transfer function corrected using $\mathrm{Gctf}^{30}$, as implemented in cryoSPARC ${ }^{31}$ (version 3.0.1). Subsequent image processing steps were performed in cryoSPARC. Initial particle selection was done using the automated blob picker on 100 micrographs, using a $100 \AA$ minimum and $150 \AA$ maximum particle diameter, to extract a total of 38,166 particles. Next, the extracted particles were subjected to one round of 2D classification, into 200 classes, from which 7 classes were selected 
and used as a template for the automatic particle picking process. A total of 3,062,337 particles were extracted from the first 6,450 micrographs, followed by a round of $2 \mathrm{D}$ classification that resulted in 200 classes. Finally, a round of 3D reconstructions and classification produced 6 classes.

A total of 3,504,800 particles were extracted from the second data-set of image-stacks, followed by a round of $2 \mathrm{D}$ classification, split into 200 classes, and a round of 3D reconstructions and classification into 3 classes. The particles from the best classes from both datasets were then joined together and subjected to one ab-initio round of 3D reconstructions split into 6 classes. A final data-set of 392,370 particles from the best 3D classes were subjected to local and global CTF refinements, followed by a 3D non-uniform refinement. The final density map was resolved to $3.25 \AA$, after map sharpening, as determined by gold-standard Fourier shell correlation using the 0.143 criterion. Local resolution estimation was performed using cryoSPARC.

\section{Model building}

An initial model was created by docking the individual complex components into the cryo-EM map using the "fit in map" routine in UCSF Chimera ${ }^{32}$. The following structures from the Protein Data Bank (PDB) were used: OTR (PDB ID: 6TPK), G $\alpha_{\mathrm{o}}$ (PDB ID: 6WWZ), G $\beta_{1} \gamma_{2}$, scFv16 (PDB ID: 6OIJ), and AVP (PDB ID: 7DW9). All initial model components were manually rebuilt in $\mathrm{Coot}^{33}$, followed by several rounds of manual real-space refinement in Coot and real-space refinement with the software package Phenix.real_space_refine from Phenix ${ }^{34}$. The quality of the models was assessed using MolProbity ${ }^{35}$ before PDB deposition. 


\section{IP1 accumulation assays}

Ligand-induced IP1 accumulation and ligand-binding experiments were measured using transiently transfected Human Embryonic Kidney (HEK) 293T/17 cells (American Type Culture Collection). The cells were cultivated in Dulbecco's modified Eagle's medium (Thermo Fisher Scientific) supplemented with penicillin $(100 \mathrm{U} / \mathrm{ml})$, streptomycin $(100 \mu \mathrm{g} / \mathrm{ml}$, MilliporeSigma), and $10 \%(\mathrm{v} / \mathrm{v})$ fetal calf serum (BioConcept) and maintained at $37^{\circ} \mathrm{C}$ in a humidified atmosphere of $5 \% \mathrm{CO}_{2}$ and $95 \%$ air. Transient transfections were performed with TransIT-293 (Mirus Bio) according to the manufacturer's instructions. Full-length OTR, truncated $\mathrm{V}_{1 \mathrm{~A}} \mathrm{R}$ (residues 1-378 with $\mathrm{C}$-terminal mutation $\mathrm{T} 378 \mathrm{~K}$ ) and mutants thereof were directly cloned into a mammalian expression vector containing an N-terminal SNAP-tag (pMC08; Cisbio). Cells were transfected and directly seeded at 7500 cells per well in poly-Dlysine-coated white 384-well plates (Greiner).

To compare IP1 accumulation a homogeneous time-resolved fluorescence (HTRF) signalling assay was performed, adapting a previously described protocol ${ }^{36}$. The cells were washed 48 hours after transfection with phosphate-buffered saline (PBS) and stimulation buffer (Cisbio) and subsequently incubated for 1 hour at $37^{\circ} \mathrm{C}$ with a concentration range of oxytocin (Psyclo Peptide Inc.) diluted in stimulation buffer. The IP1 accumulation was determined using the HTRF IP-One Kit (Cisbio) according to the manufacturer's protocol. Fluorescence intensities were measured on a Spark fluorescence plate reader (Tecan). To generate concentration-response curves, data were analysed by a three-parameter logistic equation in GraphPad Prism software (version 9.2.0).

\section{Whole-cell ligand binding assays}

Ligand-binding experiments were performed on whole cells for comparison of affinities for wild-type and receptor mutants using an HTRF binding assay as previously described ${ }^{14}$. Forty- 
eight hours post-transfection the cells were labelled with $50 \mathrm{nM}$ SNAP-Lumi4-Tb (Cisbio) in labelling buffer (20 mM Hepes (pH 7.5), $100 \mathrm{mM} \mathrm{NaCl}, 3 \mathrm{mM} \mathrm{MgCl}_{2}$, and $0.05 \%$ (w/v) bovine serum albumin (BSA)) for 1.5 hours at $37^{\circ} \mathrm{C}$. The cells were washed two times with labelling buffer and two times with assay buffer (20 mM Hepes (pH 7.5), $100 \mathrm{mM} \mathrm{KCl,} 3 \mathrm{mM} \mathrm{MgCl}$, and $0.05 \%(\mathrm{w} / \mathrm{v}) \mathrm{BSA}))$ and subsequently incubated for $1 \mathrm{~h}$ at room temperature with a concentration range of fluorescently labelled peptide HiLyte Fluor 488-Orn ${ }^{8}$ oxytocin (Eurogentec) in assay buffer. Fluorescence intensities were measured on a Spark fluorescence plate reader with an excitation wavelength of $340 \mathrm{~nm}$ and emission wavelengths of $620 \mathrm{~nm}$ and $510 \mathrm{~nm}$ for $\mathrm{Tb}^{3+}$ and the fluorophore HiLyte Fluor 488, respectively. The ratio of fluorescence resonance energy transfer (FRET) donor and acceptor fluorescence intensities was calculated $(\mathrm{F} 510 \mathrm{~nm} / \mathrm{F} 620 \mathrm{~nm})$. Nonspecific binding was determined in the presence of a 1000-fold excess of unlabelled oxytocin. Data were analysed by global fitting to a one-site saturation binding equation with the GraphPad Prism software. 


\section{References}

1. Barberis, C., Mouillac, B. \& Durroux, T. Structural bases of vasopressin/oxytocin receptor function. J. Endocrinol. 156, 223-229 (1998).

2. Donaldson, Z.R. \& Young, L.J. Oxytocin, vasopressin, and the neurogenetics of sociality. Science 322, 900-904 (2008).

3. Guastella, A.J. \& Hickie, I.B. Oxytocin treatment, circuitry, and autism: a critical review of the literature placing oxytocin into the autism context. Biol. Psychiatry 79, 234-242 (2016).

4. Neumann, I.D. \& Slattery, D.A. Oxytocin in general anxiety and social fear: a translational approach. Biol. Psychiatry 79, 213-221 (2016).

5. Pedersen, C.A. et al. Intranasal oxytocin reduces psychotic symptoms and improves Theory of Mind and social perception in schizophrenia. Schizophr. Res. 132, 50-53 (2011).

6. Shyken, J.M. \& Petrie, R.H. Oxytocin to Induce Labor. Clin. Obstet. Gynecol. 38(1995).

7. Wiśniewski, K. Design of oxytocin analogs. in Cyclic Peptide Design (ed. Goetz, G.) 235-271 (Springer New York, New York, NY, 2019).

8. Nashar, P.E., Whitfield, A.A., Mikusek, J. \& Reekie, T.A. The current status of drug discovery for the oxytocin receptor in Oxytocin: Methods and Protocols (eds. Werry, E.L., Reekie, T.A. \& Kassiou, M.) 153-174 (Springer US, New York, NY, 2022).

9. Waltenspühl, Y., Jeliazkov, J.R., Kummer, L. \& Plückthun, A. Directed evolution for high functional production and stability of a challenging $G$ protein-coupled receptor. Sci. Rep. 11, 8630 (2021).

10. Nehmé, R. et al. Mini-G proteins: Novel tools for studying GPCRs in their active conformation. PLOS ONE 12, e0175642 (2017).

11. Thom, C. et al. Structures of neurokinin 1 receptor in complex with $\mathrm{Gq}$ and Gs proteins reveal substance P binding mode and unique activation features. Sci. Adv. 7, eabk2872 (2021).

12. Busnelli, M. et al. Functional selective oxytocin-derived agonists discriminate between individual G protein family subtypes. J. Biol. Chem. 287, 3617-3629 (2012).

13. Ferrier, B.M. \& du Vigneaud, V. 9-deamidooxytocin, an analog of the hormone containing a glycine residue in place of the glycinamide residue. J. Med. Chem. 9, 5557 (1966).

14. Waltenspühl, Y., Schöppe, J., Ehrenmann, J., Kummer, L. \& Plückthun, A. Crystal structure of the human oxytocin receptor. Sci. Adv. 6, eabb5419 (2020).

15. Durroux, T. et al. Fluorescent pseudo-peptide linear vasopressin antagonists: design, synthesis, and applications. J. Med. Chem. 42, 1312-1319 (1999). 
16. Wang, L. et al. Cryo-EM structure of the AVP-vasopressin receptor 2-Gs signaling complex. Cell Ress 31, 932-934 (2021).

17. Chini, B. et al. Tyr115 is the key residue for determining agonist selectivity in the V1a vasopressin receptor. The EMBO Journal 14, 2176-2182 (1995).

18. Bous, J. et al. Cryo-electron microscopy structure of the antidiuretic hormone argininevasopressin V2 receptor signaling complex. Sci. Adv. 7, eabg5628 (2021).

19. Zhou, F. et al. Molecular basis of ligand recognition and activation of human V2 vasopressin receptor. Cell Res. 31, 929-931 (2021).

20. Wesley, V.J., Hawtin, S.R., Howard, H.C. \& Wheatley, M. Agonist-specific, highAffinity binding epitopes are contributed by an arginine in the N-terminus of the human oxytocin receptor. Biochemistry 41, 5086-5092 (2002).

21. Mobbs, J.I. et al. Structures of the human cholecystokinin 1 (CCK1) receptor bound to Gs and $\mathrm{Gq}$ mimetic proteins provide insight into mechanisms of $\mathrm{G}$ protein selectivity. PLoS Biol. 19, e3001295 (2021).

22. Hong, C. et al. Structures of active-state orexin receptor 2 rationalize peptide and smallmolecule agonist recognition and receptor activation. Nat. Commun. 12, 815 (2021).

23. Yuan, D. et al. Activation of the $\alpha 2 \mathrm{~B}$ adrenoceptor by the sedative sympatholytic dexmedetomidine. Nat. Chem. Biol. 16, 507-512 (2020).

24. Wasilko, D.J. et al. Structural basis for chemokine receptor CCR6 activation by the endogenous protein ligand CCL20. Nat. Commun. 11, 3031 (2020).

25. Maeda, S., Qu, Q., Robertson Michael, J., Skiniotis, G. \& Kobilka Brian, K. Structures of the M1 and M2 muscarinic acetylcholine receptor/G-protein complexes. Science 364, 552-557 (2019).

26. Maeda, S. et al. Development of an antibody fragment that stabilizes GPCR/G-protein complexes. Nat. Commun. 9, 3712 (2018).

27. Schöppe, J., Ehrenmann, J., Waltenspühl, Y. \& Plückthun, A. Universal platform for the generation of thermostabilized GPCRs that crystallize in LCP. Nat. Protoc. (2022).

28. Flock, T. et al. Universal allosteric mechanism for Galpha activation by GPCRs. Nature 524, 173-179 (2015).

29. Zheng, S.Q. et al. MotionCor2: anisotropic correction of beam-induced motion for improved cryo-electron microscopy. Nat. Methods 14, 331-332 (2017).

30. Zhang, K. Gctf: Real-time CTF determination and correction. J. Struct. Biol. 193, 1-12 (2016).

31. Punjani, A., Rubinstein, J.L., Fleet, D.J. \& Brubaker, M.A. cryoSPARC: algorithms for rapid unsupervised cryo-EM structure determination. Nat. Methods 14, 290-296 (2017). 
32. Pettersen, E.F. et al. UCSF Chimera-A visualization system for exploratory research and analysis. J. Comput. Chem. 25, 1605-1612 (2004).

33. Emsley, P., Lohkamp, B., Scott, W.G. \& Cowtan, K. Features and development of Coot. Acta Crystallogr. D 66, 486-501 (2010).

34. Afonine, P.V. et al. Towards automated crystallographic structure refinement with phenix.refine. Acta Crystallogr. D 68, 352-367 (2012).

35. Williams, C.J. et al. MolProbity: More and better reference data for improved all-atom structure validation. Protein Sci. 27, 293-315 (2018).

36. Ehrenmann, J. et al. High-resolution crystal structure of parathyroid hormone 1 receptor in complex with a peptide agonist. Nat. Struct. Mol. Biol. 25, 1086-1092 (2018). 


\section{Acknowledgements}

We thank the staff, in particular S. Sorrentino, of the Centre for Microscopy and Image Analysis at the University of Zurich for their support in the initial phases of the project. We further thank O. Eggenberger for assistance in protein production and I. Berger for providing us with baculovirus transfer vectors.

\section{Funding}

This work was supported by Schweizerischer Nationalfonds Grant 31003A_182334 to A.P, and by the European Research Council (810057-HighResCells to A.P. and O.M.).

\section{Author contributions}

The project was outlined by Y.W. and A.P.. Y.W., and J.E. designed the G protein and scFv constructs. Y.W. purified all proteins and prepared the complexes. Y.W, J.E. and S.V. vitrified the samples on cryo-EM grids. Y.W. and J.E. collected cryo-EM data with the help of S.V. Y.W. and S.V. processed data and refined the cryo-EM density map. J.E. and Y.W. built and refined the structure model. C.T. designed and performed the functional assays. Project management was carried out by Y.W, J.E., C.T., O.M. and A.P. The manuscript was prepared by Y.W., C.T., S.V., J.E., O.M. and A.P. All authors contributed to the final editing and approval of the manuscript. 


\section{Competing interests}

The authors declare no competing interests.

\section{Data availability}

Atomic coordinates of the OTR: $\mathrm{G}_{\mathrm{o} / \mathrm{q}}: \mathrm{OT}: \mathrm{scFv} 16$ complex have been deposited in the PDB under the accession code 7QVM. Cryo-EM maps used have been deposited in the EMDB found under code EMD-14180. 
a

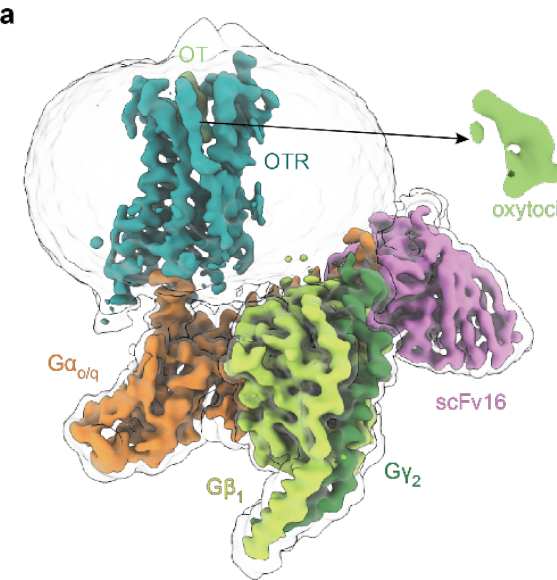

d

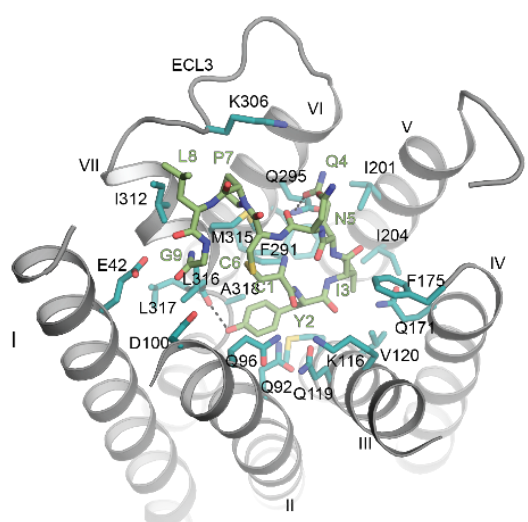

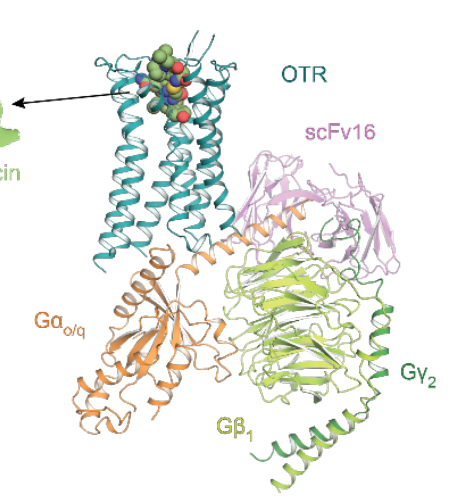

c

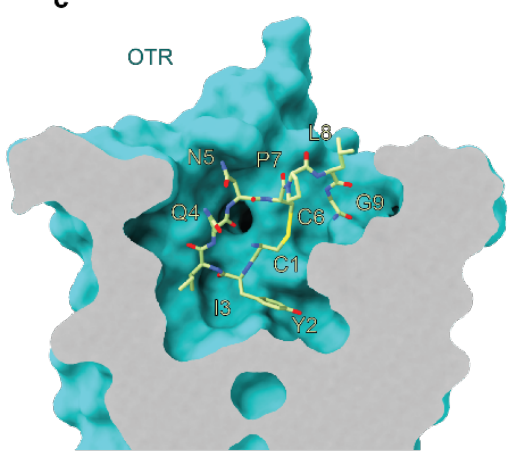

e

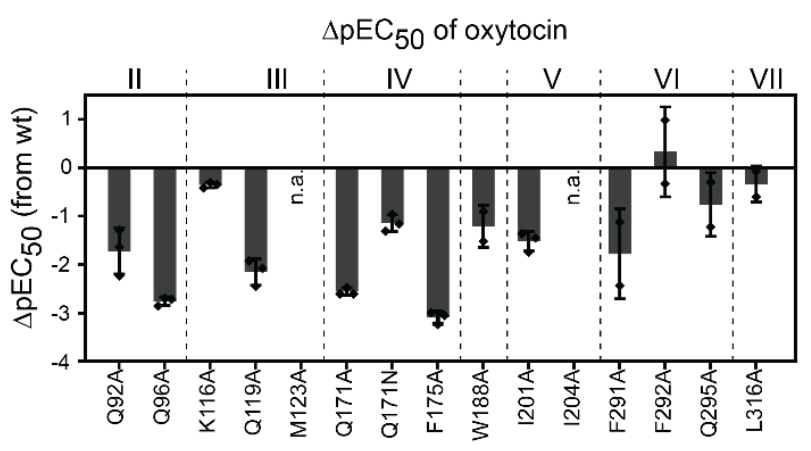

Fig. 1: Overall structure of the oxytocin bound OTR

a Cryo-EM map of the OTR:OT: $\mathrm{G}_{\mathrm{o} / \mathrm{q}}: \mathrm{ScFv} 16$ complex. b Molecular model of the OTR:OT:G $\mathrm{G}_{\mathrm{o} / \mathrm{q}}: \mathrm{scFv} 16$ complex. Ligand atoms are coloured according to atom types. c Clipped surface representation of the OT orthosteric binding pocket with OT shown as bright green sticks. d Detailed interactions of OT with OTR. e Agonist potency profiles of selected mutants. Bars represent differences in IP1 accumulation potency (mean $\Delta \mathrm{pEC}_{50} \pm$ standard deviation from at least two independent experiments in duplicates) compared to wtOTR. n.a., no activation. 

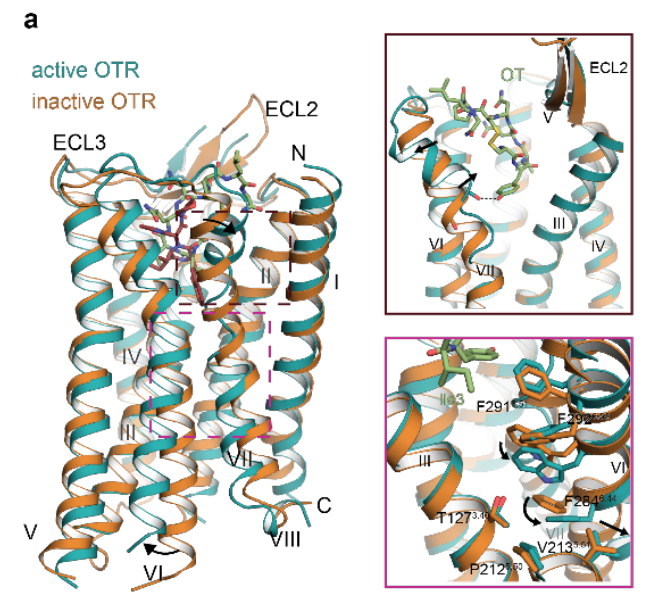

c

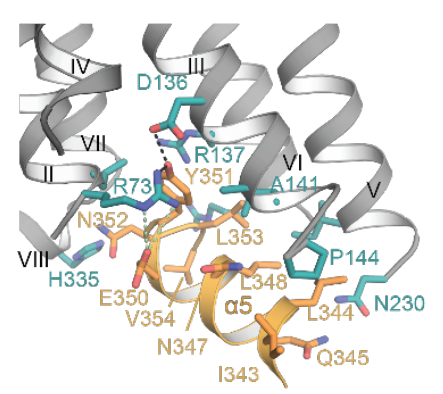

d

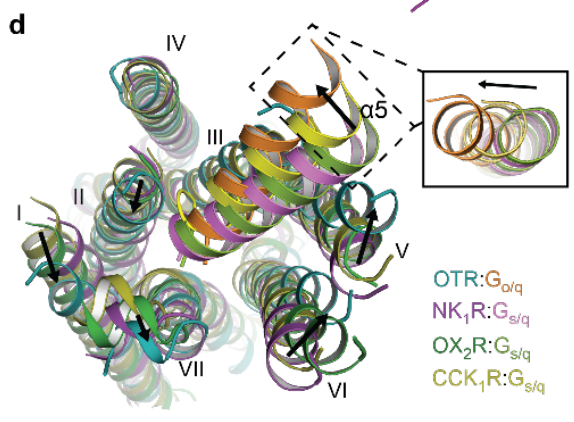

b
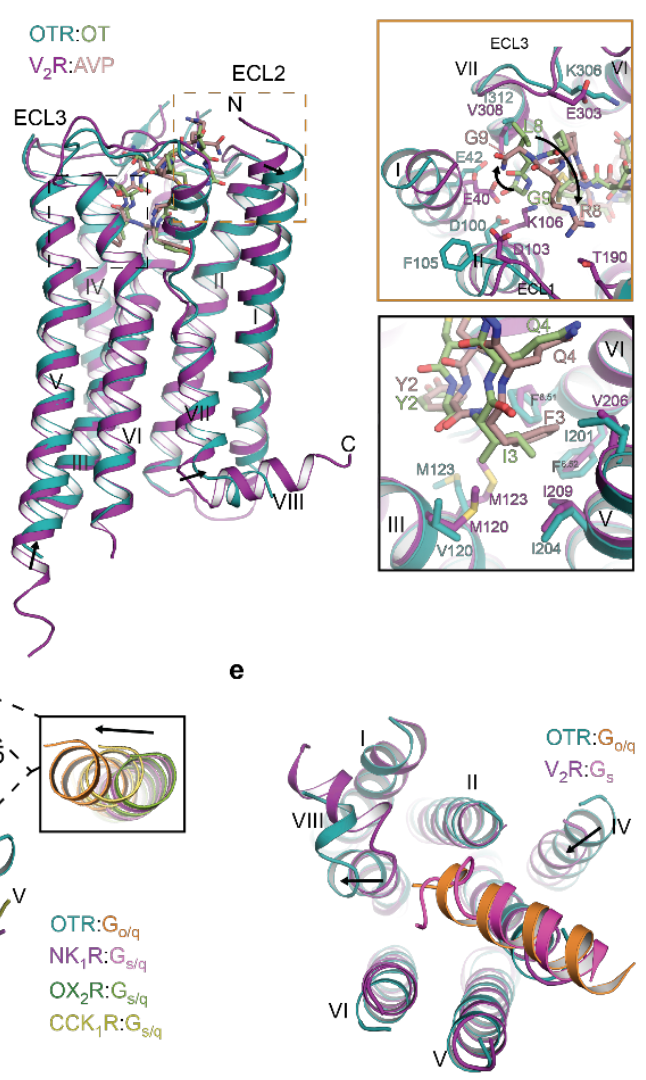

Fig. 2: Activation of OTR

a Molecular mechanism of OTR activation. Superposition of active (teal, this study [PDB 7QVM]) and inactive (orange, PDB 6TPK) OTR. b Structural superposition of OTR:OT and $\mathrm{V}_{2} \mathrm{R}: \mathrm{AVP}$ (purple, PDB 7KH0) complexes, with close-up on agonist positions 3 (bottom right) and position 8 (top right). c Detailed interactions of $\mathrm{G}_{\mathrm{q}} \alpha 5$ with OTR. d Structural comparison of OTR: $\mathrm{G}_{\mathrm{o} / \mathrm{q}}$ to $\mathrm{G}_{\mathrm{s} / \mathrm{q}}$ complexes with focus on $\mathrm{G}_{\mathrm{q}} \alpha 5$ positioning: $\mathrm{NK}_{1} \mathrm{R}: \mathrm{G}_{\mathrm{s} / \mathrm{q}}$ (purple, PDB 7P00), $\mathrm{OX}_{2} \mathrm{R}: \mathrm{G}_{\mathrm{s} / \mathrm{q}}$ (green, PDB 7L1U), and $\mathrm{CCK}_{1} \mathrm{R}: \mathrm{G}_{\mathrm{s} / \mathrm{q}}$ (yellow, PDB 7MBY). e Structural comparison of OTR: $\mathrm{G}_{\mathrm{o} / \mathrm{q}}$ and $\mathrm{V}_{2} \mathrm{R}: \mathrm{G}_{\mathrm{s}}$ (pink, PDB 7KH0) with focus on helix $\alpha 5$ positioning. 